\title{
The sources of heat generation in vibrothermography
}

\author{
Jeremy Renshaw, J ohn C. Chen, Stephen D. Holland, R. Bruce Thompson
}

\begin{abstract}
A B S T R A C T
Vibrothermography, or sonic IR, is a nondestructive evaluation technique used to find surface and near surface defects-such as cracks and delaminations - through observations of vibration-induced heat generation. This method has significant interest as an industrial inspection method, however, a lack of understanding of the fundamental physics governing the heat generation process has limited its application despite extensive theoretical, numerical simulation, and experimental work. Significant theoretical and numerical simulation work has been performed, but has yet to be rigorously verified experimentally. This paper presents experimental verification of the sources of heat generation in vibrothermography; specifically friction, plasticity, and viscoelasticity. Specific experimental evidence is presented that verifies each of these heat-generating mechanisms.
\end{abstract}

\section{Introduction}

Vibrothermography is a nondestructive evaluation technique used to find surface and near surface defects. Also known as thermosonics and sonic IR [1], vibrothermography detects and locates cracks, disbonds, and delaminations using the heat generated by these defects when they are vibrated. The generated heat diffuses away from these thermal indications and radiates from the surfaces. Radiated heat is observed and measured using an infrared (IR) camera observing the surface of the structure containing the defect indications. There is significant industrial interest in vibrothermography due to its ability to rapidly and accurately detect cracks and other defects in structures [2]; however, it has been hindered by issues such as repeatability [1], due in part, to a lack of understanding of the physics governing the heat generation process in vibrating defects.

Reifsnider et al. [3] observed heat generation at artificial delaminations in composites when vibrated. They noted that heat generation at the delaminations increased when samples were vibrated at certain resonant frequencies. Subsequently, many similar experiments have been performed on samples containing cracks and other defects $[1,4,5]$. It has been shown that regions of heat generation along a crack correspond to contacting regions in the crack and the location of these regions changes as the crack is opened with an extemal load [5,6]. Lu et al. [7] showed that increasing compressive stresses on a crack can terminate heat generation, presumably by locking crack face asperities together with static friction. Frictional rubbing between contacting asperities has generally been accepted as a source of heat generation in vibrothermography $[1,8,9]$. However, there is controversy with this claim since it has also been argued that frictional rubbing between crack faces is not responsible for heat generation or energy dissipation, but that crack heating is entirely due to interactions in the elastoplastic region of a crack [10]. Despite numerous finite element simulations and theoretical explanations, no definitive experimental validation of either theory has been presented and no single theory has been universally accepted to date to explain the source—or sources—of heat generation in vibrothermography.

This paper presents experimental evidence correlating heat generation in vibrothermography to specific heat-generating mechanisms. Crack heating in these experiments is generated via three mechanisms: first due to frictional rubbing of contacting regions (asperities) on crack faces. Second, plastic deformations, which occur when cracks begin to propagate, generate heat in the plastic zone surrounding the crack. Third, viscoelastic losses can generate significant heat in some materials and these losses are larger in regions of stress concentration [11], such as around delaminations or other defects.

\section{Theory}

Heat generation in cracks is primarily generated from one or a combination of three mechanisms: friction, plasticity, and viscoelasticity/material damping.

Spatially isolating regions of heat generation at a thermal indication provides a means to determine contributions from different heatgenerating mechanisms on the total heat generation of the indication. In cracks, frictional heat generation occurs due to rubbing of contacting regions (asperities) along crack faces. Frictional rubbing may also induce plastic deformation of rubbing asperities, evidenced by plow marks on the asperities [12-14]. Such deformations are classified here as "friction,"first because they are friction-induced and, second, to avoid confusion with plastic deformations near the crack tip associated with crack propagation and material degradation. Frictional heat generation cannot occur past the crack tips in vibrothermography since there is no surface available to rub. Crack growth, however, can cause heat generation in the plastic zone beyond a crack tip due to plastic deformations in the crack's plastic zone. Viscoelastic heating is negligible in most metals, but can be a significant heat source for polymer-based materials [3,11]. Viscoelastic heating is evidenced by bulk heating of the structure from the vibrational stress with additional localized heating at regions of stress concentration, so it is generally possible to distinguish between plasticity-induced and viscoelastic-induced heating. Measurements of vibrational stresses further aid in distinguishing between heating from plasticity, which only begins to occur above a specific threshold stress and in specific locations, and viscoelasticity, which is linearly related to the vibrational stress level at all points throughout a structure.

Thermoelastic heating and cooling can be significant in some circumstances, but can be averaged out of the heating data by setting the integration time of each IR camera frame to be many times longer than the vibration period. 
Observing heat generation of a crack while modifying its closure state (the regions of contact along the crack) also helps to determine whether friction or viscoelasticity is dominant. Both heating mechanisms depend on the local vibrational stress and strain at the defect, though frictional heating requires contact between crack faces to occur. Applying a tensile load to a crack lowers the closure stresses between crack faces and can separate crack faces from contact or, conversely, compressive stresses can lock them together. Both actions prevent frictional heating, though neither action would eliminate viscoelastic heating.

\section{Experimental procedure}

The experimental setup used for the tests performed in this study is shown in Fig. 1. A cracked sample is held in place using clamps and vibrated using a broadband piezoelectric transducer

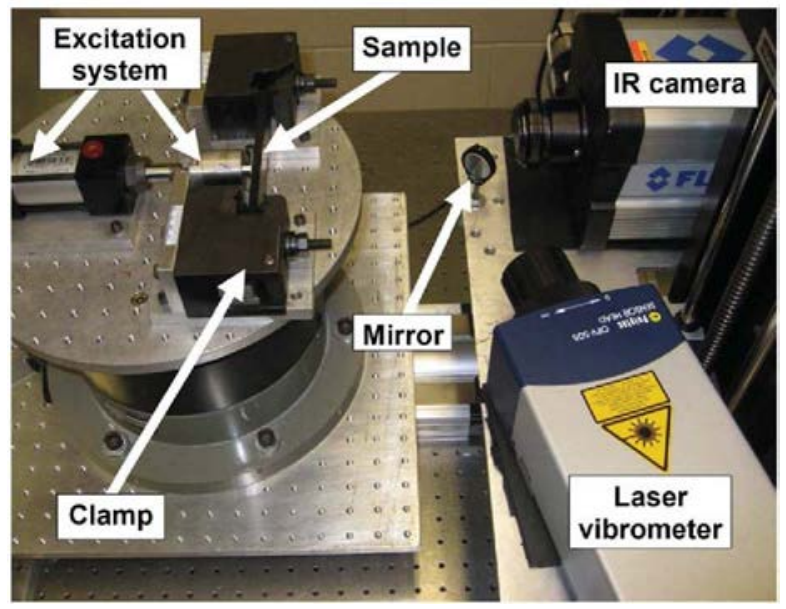

Fig. 1. Experimental setup used for exciting the test specimens and observing heat generation.

excitation system. Samples are vibrated at resonance to improve energy coupling into the sample and more effectively generate heat at defects. A laser vibrometer is used to measure the frequencies and amplitudes (velocities) of vibration excited in the samples through the Doppler shift in the laser beam after it is reflected off of the sample surface and back to the laser vibrometer. Typical vibrational frequencies used are in the $20-40 \mathrm{kHz}$ range. An IR camera is used to measure surface heating of indications [15]. Sequences of calibrated IR images are recorded for subsequent processing to extract heat generation and diffusion information that can be related back to specific heat generation sources.

The vibrational stresses used for most experiments were small in comparison to the fatigue limit of the material (i.e. less than $40 \%$ of the materials fatigue limit) to avoid causing additional damage to the specimen, such as crack growth which has been observed at high vibrational stress levels [16].

After testing was completed on each sample, the cracks were observed and their lengths were carefully measured to check whether crack growth had occurred or not. After a surface examination, the cracks were broken open to observe the rubbing crack faces using optical and scanning electron microscopy to check for friction-induced damage to the rubbing surfaces.

\section{Results}

\subsection{Frictional heating}

Previous work [5-7] provides some evidence that friction appears to be a source of vibrothermographic heating in metals since high closure stresses on crack faces can terminate heat generation, ... heat generation. However, external ... external static stresses would not have a significant effect on heat generation of a crack were viscoelasticity or plasticity primarily responsible for heat generation in these cases.

Fig. 2 shows a hypothetical schematic of how heat is generated by friction in a semielliptical surface crack. View A in Fig. 2 shows heat generation due to vibration at the surface of a semielliptical crack in titanium (Ti 6-4) with the crack length (dotted line) superimposed on top of the heating profile. View B in Fig. 2 shows a schematic side view of the semielliptical crack, correlating to the observed heat generation in View A, including open, heating, and locked asperity regions as well as the plastic zone past the crack tips. The heating profile in View A shows that heat is not generated near the crack tips in this crack, but along the crack 


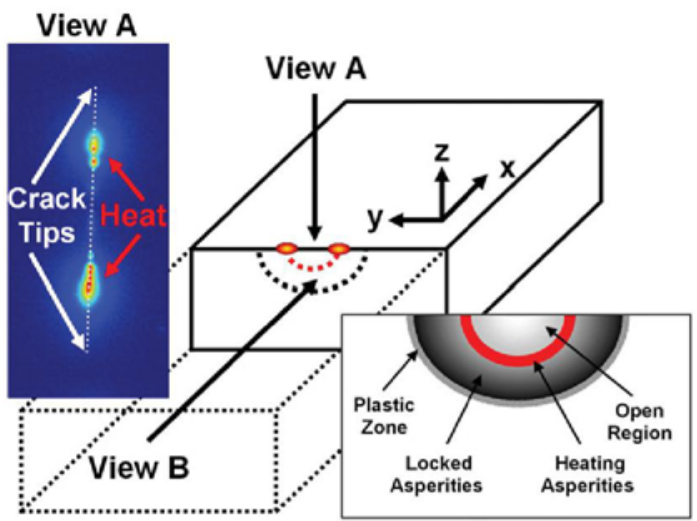

View B

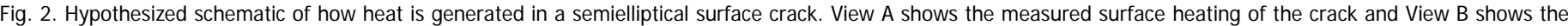

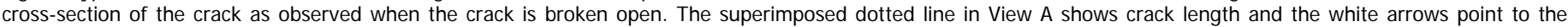
crack tips.

faces and demonstrates that, in this crack, frictional heating is the source of the observed heat generation. Additional experimental evidence is provided by Renshaw et al. [5] where varying static tensile stresses were applied to vibrating cracks, altering the regions of heat generation as the closure state of the crack changed. Similarly, Lu et al. [7] showed that high compressive stresses can lock asperities together and prevent heat generation. Heat generation from plastic deformations or viscoelasticity would not be affected by these external stresses.

Figs. 3 and 4 show IR crack heating and microscopy images for cracks in titanium (Ti 6-4) samples. These cracks were grown to a

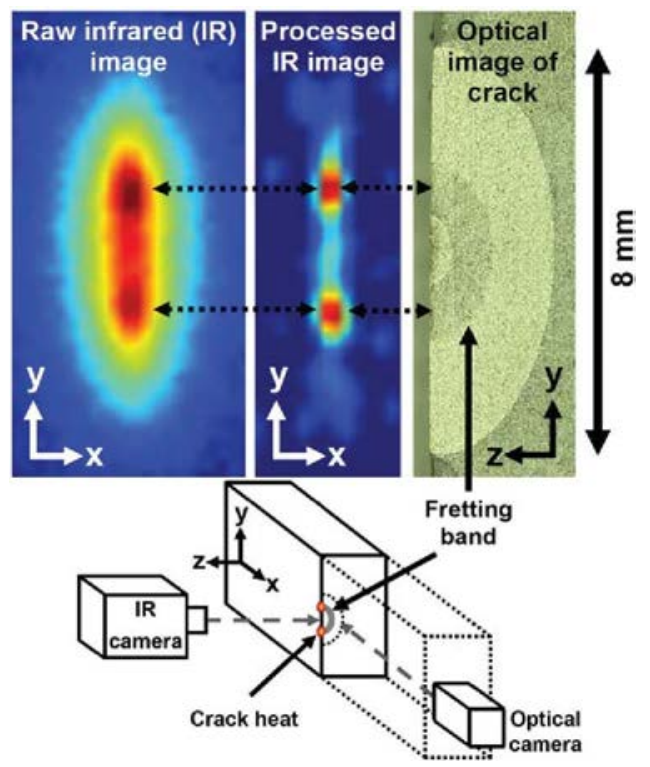

Fig. 3. Correlation of frictional heat generation to crack face fretting in titanium (Ti 6-4), (top,left) raw heating data after $1.0 \mathrm{~s}$ of excitation; (top,center) processed heating data isolating regions of heat generation; (top,right) fretting observed on crack faces; and (bottom) a schematic of the crack and sample geometry. 


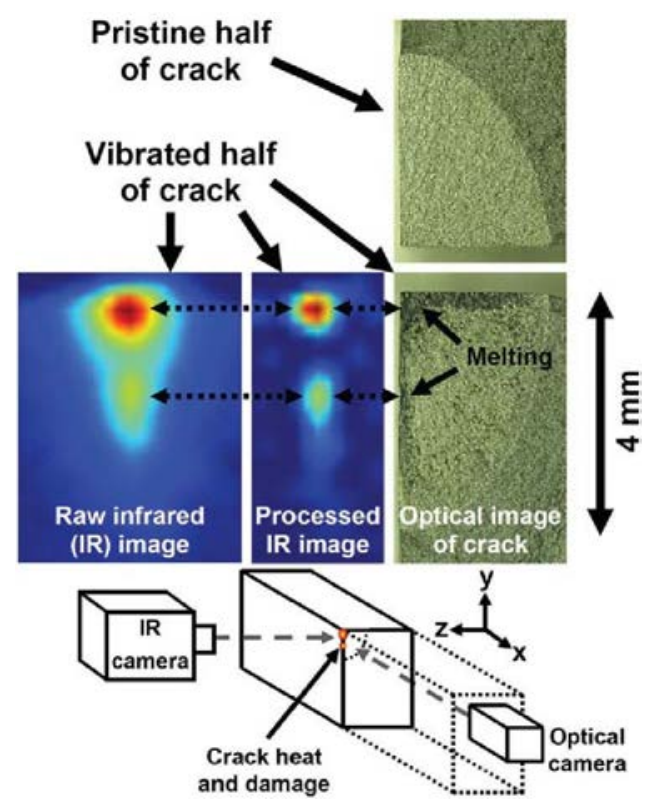

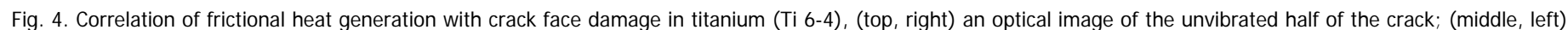

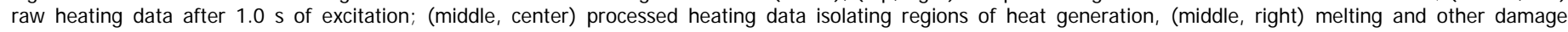
observed on the crack faces; and (bottom) a schematic of the crack and sample geometry.

length of $8.0 \mathrm{~mm}$ in three-point bending with an R-ratio ( $\mathrm{min} / \mathrm{max}$ stress) of 0.5 and maximum stress of $772 \mathrm{MPa}$. Regions of heat generation (in vibrothermography) correlate exactly to regions where fracture surface damage was observed when the crack was broken open and observed.

If frictional rubbing occurs, it can cause modifications (damage) to rubbing asperities on surfaces such as fretting, plastic deformation, melting, etc. [12,14]. Figs. 3 and 4 show a correlation between the heat generated from two cracks and the damaged regions on the crack faces caused by intense frictional rubbing. An image processing algorithm, based on the physics of heat conduction [17], was used to eliminate effects of heat diffusion and isolate regions of heat generation on the sample surface by taking a fittered, second-order spatial derivative and first-order time derivative of a series of calibrated surface temperature measurements from the IR camera. These images are calibrated by measuring an object of known length, such as a ruler, in the field of view of the camera at the location of the crack. Thus, Figs. 3 and 4 compare regions of heat generation on the sample surface to regions within the crack that were damaged due to frictional rubbing as a result of the vibration. The crack in Fig. 3 sustained fretting damage in a band that mirrors the schematic presented in Fig. 2, providing evidence that the schematic is accurate for this particular crack geometry.

Once grown to a length of $8.0 \mathrm{~mm}$, the crack in Fig. 4 was sliced in half. One half, shown at the top of Fig. 4, was kept as a pristine reference sample and the other half, shown directly below the pristine half in Fig. 4, was vibrated at resonance to generate heat and compare against the pristine reference half for evidence of frictional rubbing. The reduced cross-section and additional free surface of the crack in Fig. 4 allowed for intense vibrations to be applied, which generated sufficient frictional heat to cause oxidation and melting on the rubbing crack face asperities. These results clearly show that friction is a mechanism of heat generation in vibrothermography.

\subsection{Plasticity-induced heat generation}

Planar cracks were grown in 2024 aluminum eccentrically loaded single edge tension (ESET) samples as described in Chen et al. [16]. An annealing heat treatment was used to relieve closure stresses along the cracks. These cracked samples were then loaded into the mounting apparatus and vibrated using an ultrasonic welder. The welder can provide high vibrational amplitudes at a frequency of $20 \mathrm{kHz}$. Due to the relaxation of the closure stresses and the high vibrational stresses applied to the aluminum samples, the cracks began to grow [16], meaning that the area ahead of the crack tip was being plastically deformed and would be expected to be generating heat.

The left side of Fig. 5 shows an image of the heat generation of a propagating crack in an aluminum sample. Image processing was again used to isolate regions of heat generation in the sample, shown on the right side of Fig. 5 . Fig. 5 also shows some heat generation along the crack and two distinct regions of significant

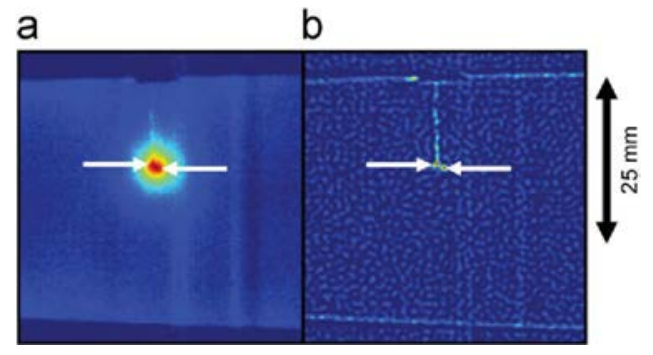

Fig. 5. (a) Raw infrared (IR) heating data compared to (b) processed IR data isolating regions of heat generation along the crack and at the crack tips. 


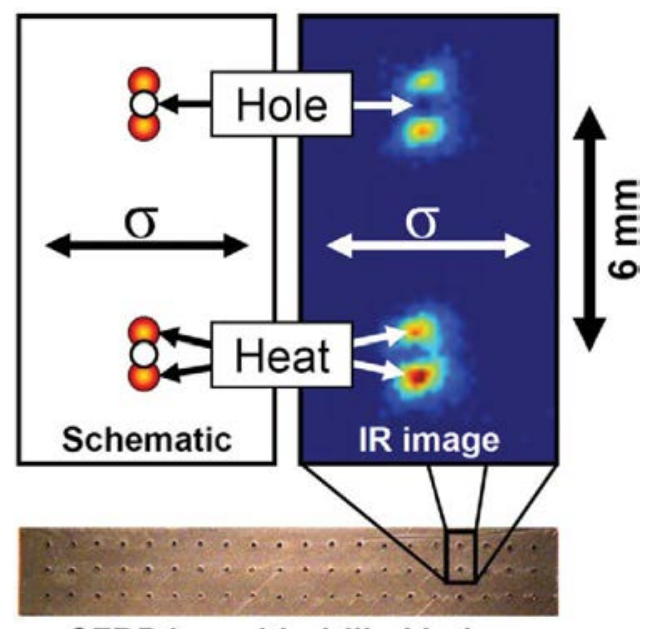

CFRP bar with drilled holes

Fig. 6. (bottom) The CFRP sample containing an array of drilled holes, (left) schematic of a CFRP sample containing drilled holes with arrows showing the direction of applied stress, and (right) observed infrared heating in the bar due to viscoelasticity and the stress concentration at the holes after $1.0 \mathrm{~s}$ of excitation.

heat generation (indicated by white arrows) near the crack tip. There are two primary regions of heat generation due to branching in the crack as it grew, the left heating region correlates to a branch in the crack that did not continue to grow while the right branch continued to grow, indicated by the heating region on the center-right side of Fig. 5, and indicated by the arrow on the right side of the figure. Heat generation at this location occurred more than $0.5 \mathrm{~mm}$ past the crack tip where plastic deformations were occurring, giving strong evidence of plasticity-induced heat generation.

It is likely that some heat near the crack tip resulted from friction during the portion of the vibration cycle where the crack faces were in contact, however, the majority of the heat generated in this experiment appears to have been the result of plastic deformations past the crack tip.

\subsection{Viscoelastic heating}

Viscoelastic heating of simulated delaminations has been observed in polymer composites by Reifsnider et al. [3]. They report that viscoelasticity is a dominant heat generation mechanisms in polymer composites. Additional viscoelastic heating is present in the vicinity of a defect due to the high stress concentrations around such defects when they are vibrated intensely, as in vibrothermographic studies. Fig. 6 shows viscoelastic heating in a carbon fiber reinforced composite (CFRP) measured using an infrared camera. Holes were drilled in the bar shown at the bottom of Fig. 6 to generate stress concentrations primarily above and below the holes when vibrated in the direction indicated in Fig. 6. Applied vibrational stresses were kept low to avoid plastic deformation. When the sample containing the drilled holes was vibrated, the stress concentrations around the holes generated heat above the baseline heating of the excited resonance in a pattern consistent with the expected stress concentration around a stressed hole, as shown in the IR heating image in Fig. 6.

\section{Conclusions}

This paper presents conclusive experimental evidence of the sources of heat generation in vibrothermography. These results show that the primary sources of heat generation in a vibrating crack include frictional rubbing, plastic deformations, and viscoelasticity in and around defects depending on the material, type of defect, and the applied vibrational stress level.

Frictional rubbing occurs, especially in cracks, and is evidenced by alterations or damage to rubbing crack faces. Plastic deformations in the plastic zone of a propagating crack may also occur at high vibrational stress levels and will generate heat in addition to friction, especially in regions beyond the crack tip. Viscoelastic heating can also occur depending on the material and is related to the vibrated material's properties and level of applied vibrational stress. Viscoelastic heating is increased in regions of stress concentration and does not require a rubbing interface to generate heat.

Improved understanding of the sources of heat generation in vibrothermography will help to design robust testing procedures to implement vibrothermography in industry as well as improve issues with experimental repeatability. This paper has shown that multiple heat-generating sources must be taken into account for successful implementation of vibrothermography as a nondestructive evaluation technique.

\section{Acknowledgements}

This material is based upon work supported by the Air Force Research Laboratory under Contract No. FA8650-04-C-5228 at lowa State University Center for NDE.

\section{References}

[1] Morbidini M, Cawley P, Barden T, Almond D, Duffour P. Prediction of the thermosonic signal from fatigue cracks in metals using vibration damping measurements. J ournal of Applied Physics 2006; 100:104905.

[2] DiMambro J, Ashbaugh DM, Nelson CL, Spencer FW. Sonic infrared (IR) imaging and fluorescent penetrant inspection probability of detection (POD) comparison. In: Thompson DO, Chimenti DE, editors. Review of progress in quantitative nondestructive evaluation, vol. 26A; 2007. p. 463-70. 


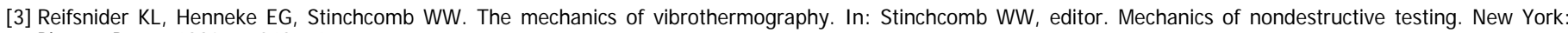
Plenum Press; 1980. p. 249-76.

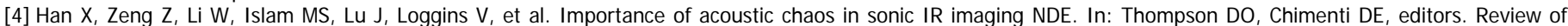
progress in quantitative nondestructive evaluation, vol. 23A; 2004. p. 496-500.

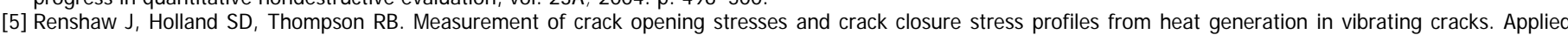
Physics Letters 2008;93(8).

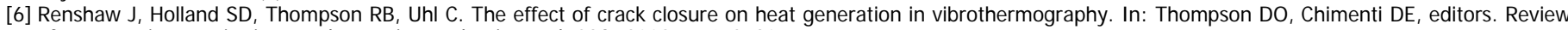
of progress in quantitative nondestructive evaluation, vol. 28A; 2009. p. 473-80.

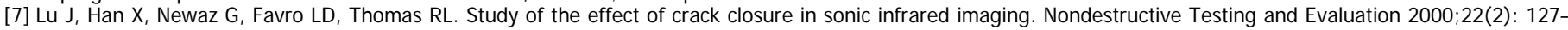
135 .

[8] Shepherd SM, Ahmed T, Lhota JR. Experimental considerations in vibrothermography. In: Proceedings of SPIE Thermosense; 2004.

[9] Zhang W, Testa RB. Closure effects on fatigue crack detection. ASCE J ournal of Engineering Mechanics 1999; 125(10): 1125-32.

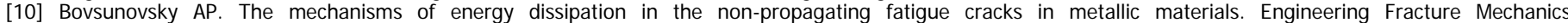
2004; 71:2271-81.

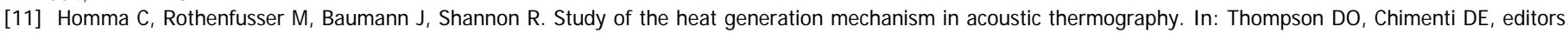
Review of progress in quantitative nondestructive evaluation, vol. 25. Melville, NY: American Institute of Physics; 2006. p. $566-73$.

[12] Bowden FP, Tabor D. The friction and lubrication of solids. Oxford University Press; 1950.

[13] Blau PJ. Friction science and technology. In: 2nd ed. New York: CRC Press; 2009. p. 119-26.

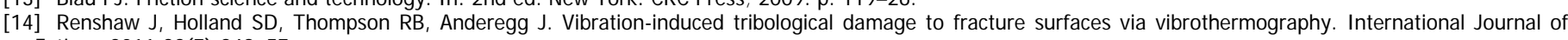
Fatigue 2011;33(7):849-57.

[15] Renshaw J, Holland SD, Barnard DJ. Viscous material-filled synthetic defects for vibrothermography. NDT\&E International 2009;42(8).

[16] Chen J C, Kephart J, Lick K, Riddell WT. Crack growth induced by sonic IR inspection. Nondestructive Testing and Evaluation $2007 ; 22(2): 83-92$.

[17] Holland SD, Renshaw J. Physics-based infrared image enhancement for thermography. NDT\&E International 2010;43(5) 